\title{
Performance evaluation of single stage diaphragmless vertical gas gun for nitrogen and helium gas propellants
}

\author{
Peter A. GARDINER*, Yuya EGAWA* and Keiko WATANABE** \\ *Department of Advanced Mechanical Engineering and Robotics, Ritsumeikan University \\ 1-1-1 Noji-higashi, Kusatsu, Shiga 525-8577, Japan \\ E-mail: rm0030pk@ed.ritsumei.ac.jp \\ ${ }^{*}$ Department of Mechanical Engineering, Ritsumeikan University \\ 1-1-1 Noji-higashi, Kusatsu, Shiga 525-8577, Japan
}

Received 6 May 2016

\begin{abstract}
The main difficulty in conducting a high velocity experiment with a projectile accelerator is predicting the projectile velocity with great accuracy. This is of crucial importance in order to keep experimental conditions constant. During the design process of the single stage diaphragmless vertical gas gun installed at the Impact Engineering Laboratory in Ritsumeikan University, a theoretical formula proposed by Seigel was used to predict the projectile velocity for various ranges of gas pressure. However, the complexity of projectile dynamics such as frictional effects between the projectile and the launch tube, the aerodynamics of the projectile, temperature effects and pressure disturbances occurring during projectile acceleration are factors excluded from the theoretical formula, making it challenging to predict the actual velocity of the projectile. Hence, it was necessary to conduct a performance evaluation of single stage diaphragmless vertical gas gun. For the experiment, flat projectiles were used consisting of a brass impactor and a high-density polyethylene sabot. A neodymium magnet was placed inside the sabot for velocity measurement using Magnet-Coil method, and nitrogen and helium were selected as gas propellants. The experimental results were carefully analyzed, and found to show a similar trend with the theoretical solution. Fitting parameters were then introduced to make the final adjustment to the theoretical formula, showing good agreement with the experimental results.
\end{abstract}

Key words : Gas gun, Performance evaluation, Projectile velocity, Theoretical prediction, Equation of motion

\section{Introduction}

In the field of impact engineering, projectile accelerators are frequently used to simulate hypervelocity impact phenomena. Gas guns, powder guns, and two-stage light gas guns are common types of projectile accelerators, selected based on the target velocity required for the experiment. Powder guns and two-stage light gas guns can achieve higher muzzle velocities than gas guns, but require time-consuming setup and cleanup processes, whereas a typical gas gun requires less setup and cleanup time per shot, providing an efficient and cost-effective testing method.

Many attempts have been made to predict and simulate a projectile accelerator's performance during the design process. Seigel (1965) introduced the concept of maintaining a constant base pressure for the projectile, referring to the most ideal situation for projectile acceleration. However, in practice, maintaining a constant pressure behind the accelerating projectile is highly problematic. Seigel offers a clear explanation of this mechanism based on the assumption that gas pressure is made up of infinite layers, leading to a theoretical solution for calculating the projectile velocity. This theoretical approach has become a well-known solution, based primarily on two fundamental parameters: the specific heat ratio of the gases and the speed of sound of gases. Mueller and Fernando (1991) investigated the dynamics of a projectile from a two-stage light gas gun, by examining the effects of forces acting between the sabot and the launch tube. Their main focus was the development of a numerical representation of dynamic stress for use in simulations, and they demonstrated the feasibility of the proposed approach. Moradi and Khodadadiyan (2011) studied the effects of real gas behaviour in a single-stage gas gun by applying the Noble-Able equation and several numerical 
solution techniques. The results showed that the projectile muzzle velocity was lower with the real gas model compared with velocities calculated using the ideal gas model, and the error between the two models gradually increased as the distance travelled by the projectile inside the launch tube increased. Furthermore, Rahner et al. (2014) conducted a numerical evaluation of the gas gun using Seigel's proposed model, to determine the most efficient gas gun for different purposes. Their paper focused on the limits and tradeoffs to be considered when designing the gas gun. Recently, at the Ernst Mach Institute, Putzar and Schaefer (2015) designed a new two-stage light gas gun in which two pistons are placed inside two separate pump tubes, instead of one piston in one pump tube. The launch delays between the two pistons are carefully adjusted to maintain the maximum instantaneous projectile base pressure for as long as possible.

For impact engineers, the fundamental requirement for a reliable hypervelocity accelerator is to have guaranteed performance in achieving the required projectile velocity with great accuracy. Several works presented above focus on optimizing the design of a typical projectile accelerator; however, these have not focused on analyzing the projectile velocity acquired from the projectile accelerator, and in particular, these have not attempted to predict or describe the accuracy with which the projectile could be launched at a desired velocity. In theory, it may be possible to predict the velocity of the projectile; however, these approaches are limited because of the difficulty of considering every aspect of projectile dynamics in calculations, from the frictional effects between the projectile and the launch tube, to the aerodynamics of the projectile, as well as the temperature effects and pressure disturbances that occur during projectile acceleration. Therefore, this paper aims to provide evidence that the projectile accelerator was correctly designed by achieving the maximum target velocity of approximately $500 \mathrm{~m} / \mathrm{s}$, and to present a method of predicting the projectile velocity by adjusting the theoretical formula to account for the experimental results.

\section{Gas gun characteristics}

A schematic diagram of a typical single stage gas/powder gun and a two-stage light gas gun are shown in Fig. 1 and Fig. 2, respectively.

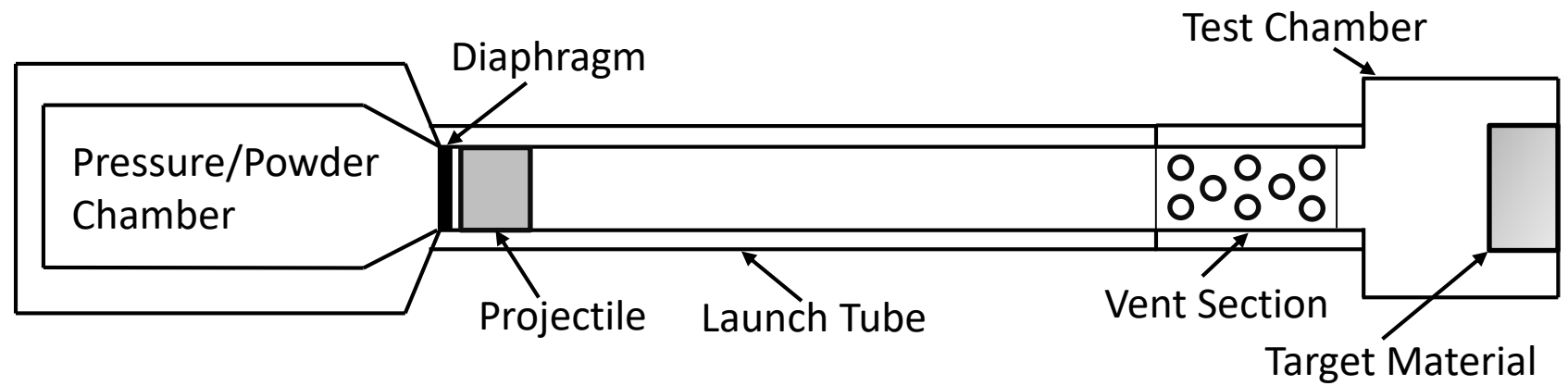

Fig. 1 Schematic diagram of a typical single stage gas/powder gun.

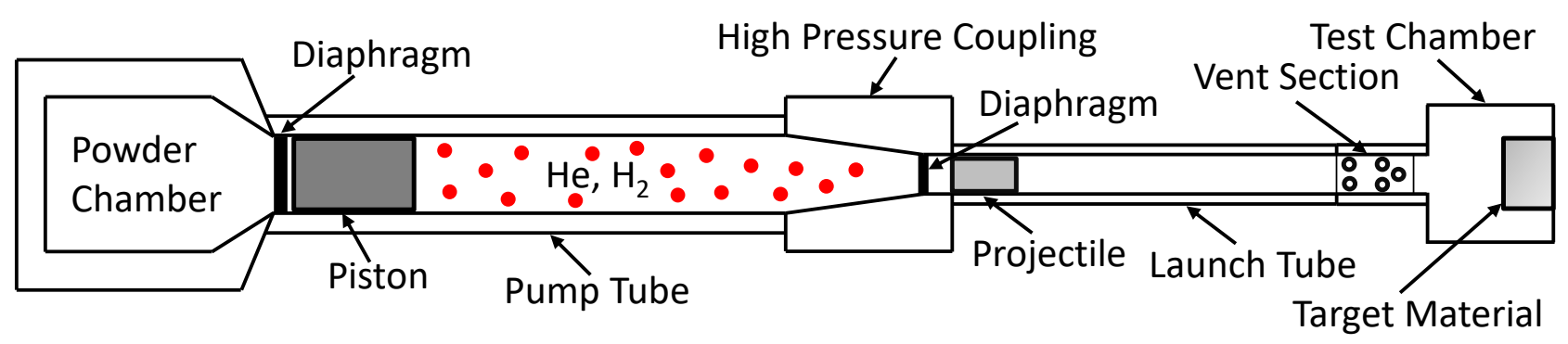

Fig. 2 Schematic diagram of a typical two-stage light gas gun. 
The single stage gas/powder gun shown in Fig. 1 operates by first filling the pressure/powder chamber with high-pressure gas. For gas guns, gases such as nitrogen or helium are used, whereas for powder guns, gunpowder is ignited to create immense pressure inside the chamber. Once the desired pressure is attained, the diaphragm between the pressure/powder chamber and the launch tube will rupture, allowing the flow of gas towards the projectile for acceleration. For the two-stage light gas gun shown in Fig. 2, gunpowder is ignited inside the powder chamber, causing the diaphragm between the chamber and the pump tube to rupture, which in turn allows the acceleration of the piston that compresses the light gas at very high velocities. This helps to increase the speed of sound of gases because the gas temperatures and pressures rise very rapidly. The compressed gas then ruptures the second diaphragm placed between the high-pressure coupling section and the launch tube, and the projectile is finally accelerated. Finally, the projectile enters the test chamber and makes contact with the target material.

At Ritsumeikan University's Impact Engineering Laboratory (Shiga, Japan), a single stage gas gun was designed to investigate the impact penetration phenomena of impacts in sand, known for showing fluid-like behaviour. When targeting a material that exhibits fluidity with a projectile accelerator, difficulties arise in maintaining the horizontal position of the target material. A special container may be used to maintain the desired position of the target material; however, once the projectile starts penetrating the target, the material will leak from its container, causing further difficulties. Furthermore, the placement of a diaphragm behind the projectile can cause additional problems. Once the diaphragm is ruptured, the remaining diaphragm material falls with the projectile, which results in affecting the target material. To overcome these problems, a single stage diaphragmless vertical gas gun was designed. The gun was set to achieve projectile muzzle velocities of up to approximately $500 \mathrm{~m} / \mathrm{s}$.

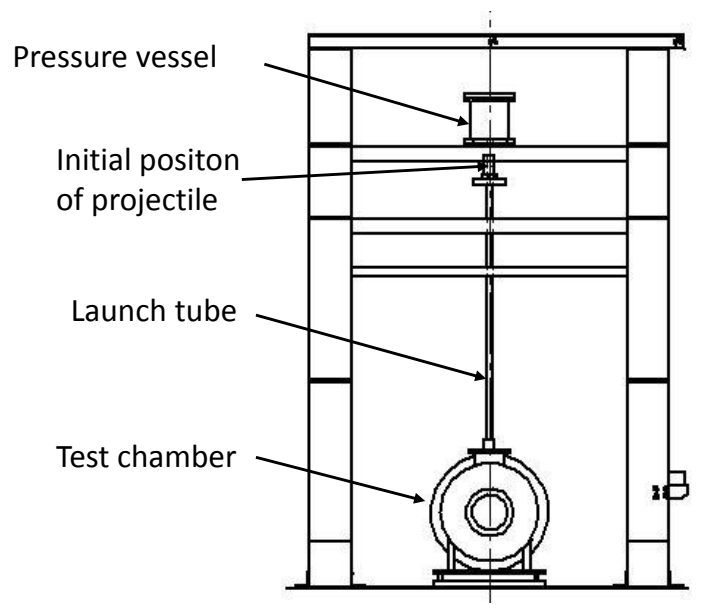

Fig. 3 Schematic diagram of gas gun.

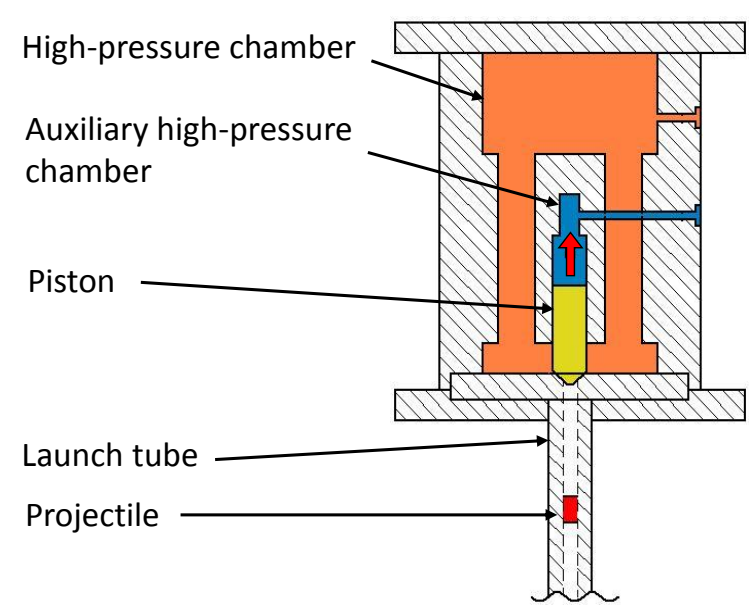

Fig. 4 Schematic diagram of pressure vessel.

As shown in Fig. 3, the gas gun installed at the Impact Engineering Laboratory consists of three main parts: the pressure vessel, launch tube, and test chamber. Fig. 4 shows the pressure vessel in detail, which was divided into two pressure chambers with a piston placed in between them. First, the auxiliary high-pressure chamber is pressurized with gas, which forces the piston to drop, closing the route between the high-pressure chamber and the launch tube. Next, gas is sent into the high-pressure chamber until the pressure desired for projectile acceleration is attained. The launch tube will remain shut, as long as the pressure inside the auxiliary high-pressure chamber is set greater than the pressure inside the high-pressure chamber. Once the pressures in both chambers reach their carefully predetermined values, the gas inside the auxiliary high-pressure chamber is released, causing the piston to rise because of the pressure differences between the chambers. This opens the route between the high-pressure chamber and the launch tube, triggering the onset of projectile acceleration. This new feature lowers operational costs and does not interfere with the target material because no diaphragm is required. Table 1 shows the gas gun parameters, including the parameters of the nitrogen and helium gases (Thompson, 1972). 
Table 1 Gas gun parameters.

\begin{tabular}{c|c}
\hline \hline Projectile mass: $m[\mathrm{~g}]$ & 16 \\
\hline Cross-sectional area of launch tube: $A\left[\mathrm{~m}^{2}\right]$ & $1.77 \times 10^{-4}$ \\
\hline Gas pressure in high-pressure vessel: $P_{0}[\mathrm{MPa}]$ & $1,2,3,5,7,9$ \\
\hline Length of launch tube: $x_{\max }[\mathrm{m}]$ & 2 \\
\hline Gas specific heat ratio: $\gamma\left(\mathrm{N}_{2}, 293.15 \mathrm{~K}\right)$ & 1.401 \\
\hline Speed of sound: $a_{0}[\mathrm{~m} / \mathrm{s}]\left(\mathrm{N}_{2}, 293.15 \mathrm{~K}\right)$ & 349.1 \\
\hline Gas specific heat ratio: $\gamma(\mathrm{He}, 293.15 \mathrm{~K})$ & 1.667 \\
\hline Speed of sound: $a_{0}[\mathrm{~m} / \mathrm{s}](\mathrm{He}, 293.15 \mathrm{~K})$ & 1007.4 \\
\hline
\end{tabular}

\section{Theoretical projectile velocity}

Seigel's theory was employed in the design of the single stage diaphragmless vertical gas gun (Seigel, 1965). The equation of motion for the projectile is given below.

$$
m \frac{d v}{d t}=P_{0} A\left\{1-\frac{v(\gamma-1)}{2 a_{0}}\right\}^{\frac{2 \gamma}{\gamma-1}}
$$

In Eq. (1), $P_{0}$ is the gas pressure, $a_{0}$ is the speed of sound of gases, $\gamma$ is the gas's specific heat ratio, $v$ is the projectile velocity, $A$ is the cross-sectional area of the launch tube, and $m$ is the projectile mass. Solving Eq. (1) yields Eq. (2), an expression for projectile velocity $v$ as a function of the distance travelled by the projectile in the launch tube $x$.

$$
x=\frac{2 m a_{0}^{2}}{P_{0} A(\gamma+1)}\left[\frac{\frac{2}{\gamma-1}-\frac{\gamma+1}{\gamma-1}\left\{1-\frac{v(\gamma-1)}{2 a_{0}}\right\}}{\left\{1-\frac{v(\gamma-1)}{2 a_{0}}\right\}^{\frac{\gamma+1}{\gamma-1}}}+1\right]
$$

The projectile velocity can be solved numerically using computational software. The theoretical projectile velocities for nitrogen and helium gases are shown in Fig. 5. In the most ideal situation, the maximum projectile velocity is achieved when the projectile base pressure reaches zero, (as for infinite launch tube lengths). After the projectile reaches its maximum velocity, it will not experience further acceleration; thus, by substituting the condition $d v / d t=0$ in Eq. (1), the projectile's theoretical maximum velocity, also known as the escape velocity, can be derived as shown in Eq. (3) (Doolan, 2001).

$$
v_{\max }=v_{e s c}=\frac{2 a_{0}}{\gamma-1}
$$


Equation (3) shows that the projectile's escape velocity depends only on two parameters: the specific heat ratio of the gases and the speed of sound of gases. A lower specific heat ratio and a higher speed of sound are preferred for higher projectile velocities; however, the speed of sound has a greater influence than the specific heat ratio, a trend that also applies to Eq. (1).

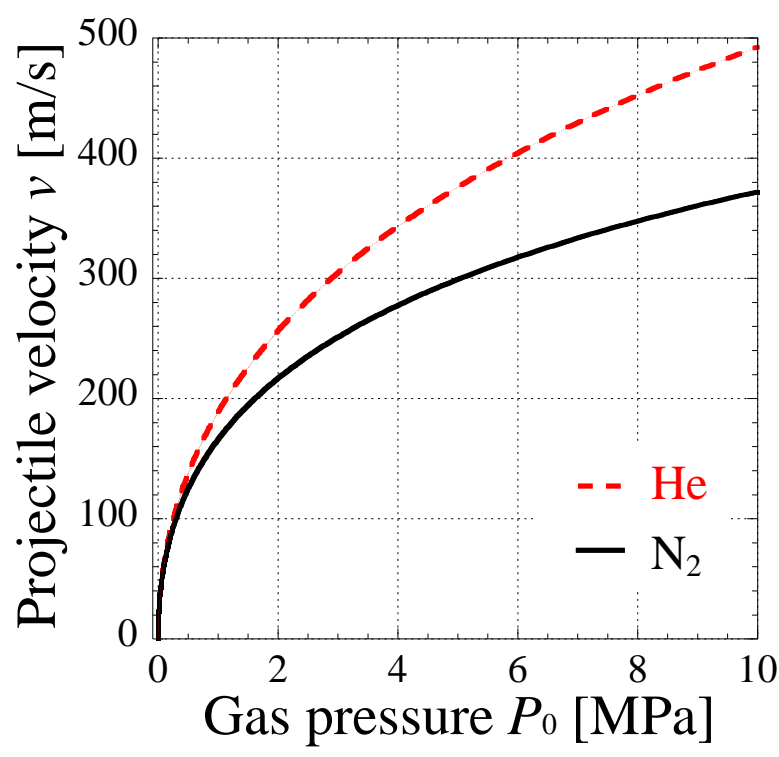

Fig. 5 Theoretical projectile velocity.

\section{Experimental procedure}

The performance evaluation was conducted with a flat projectile with mass of $16 \mathrm{~g}$, consisting of three parts as shown in Fig. 6. The diameter of the brass impactor was designed to be smaller than the diameter of the high-density polyethylene sabot to avoid internal damage of the launch tube. Furthermore, a neodymium magnet was placed behind the projectile to measure the velocity of the projectile by Magnet-Coil method; the velocity measuring device is shown in Fig. 7. Electrical signals are generated as the projectile passes through the coils, producing three sets of waveforms on the oscilloscope. Velocity is calculated by dividing the distance between the coils ( $20 \mathrm{~mm}$ ) by the time difference between the waveforms. The parameters considered in this performance evaluation were gas pressures of 1, 2, 3, 5, 7, and $9 \mathrm{MPa}$ of nitrogen and helium gas and projectile sabot diameters in the range from 14.94 to $14.99 \mathrm{~mm}$, in $0.01 \mathrm{~mm}$ intervals. Several shots were executed with each sabot diameter for a given range of gas pressure. The thermal expansion effects of the sabot can be neglected because experiments were conducted at room temperature.

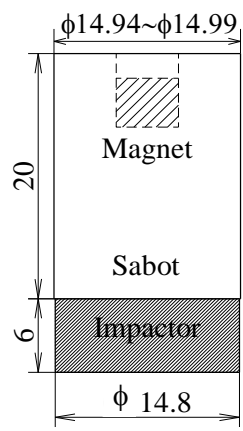

Unit: $\mathrm{mm}$

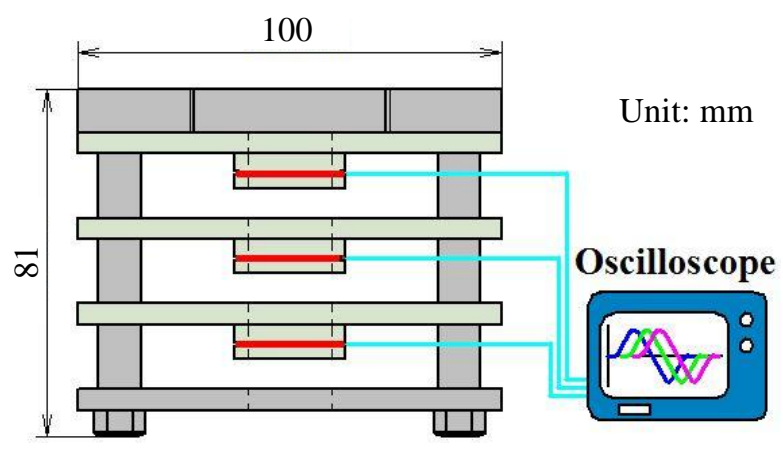

Fig. 6 Projectile specifications.

Fig. 7 Device for measuring projectile velocity. 


\section{Results and discussion}

A total of 131 shots were executed for the experiment, and the results for both nitrogen gas and helium gas are presented in Figs. 8-13.
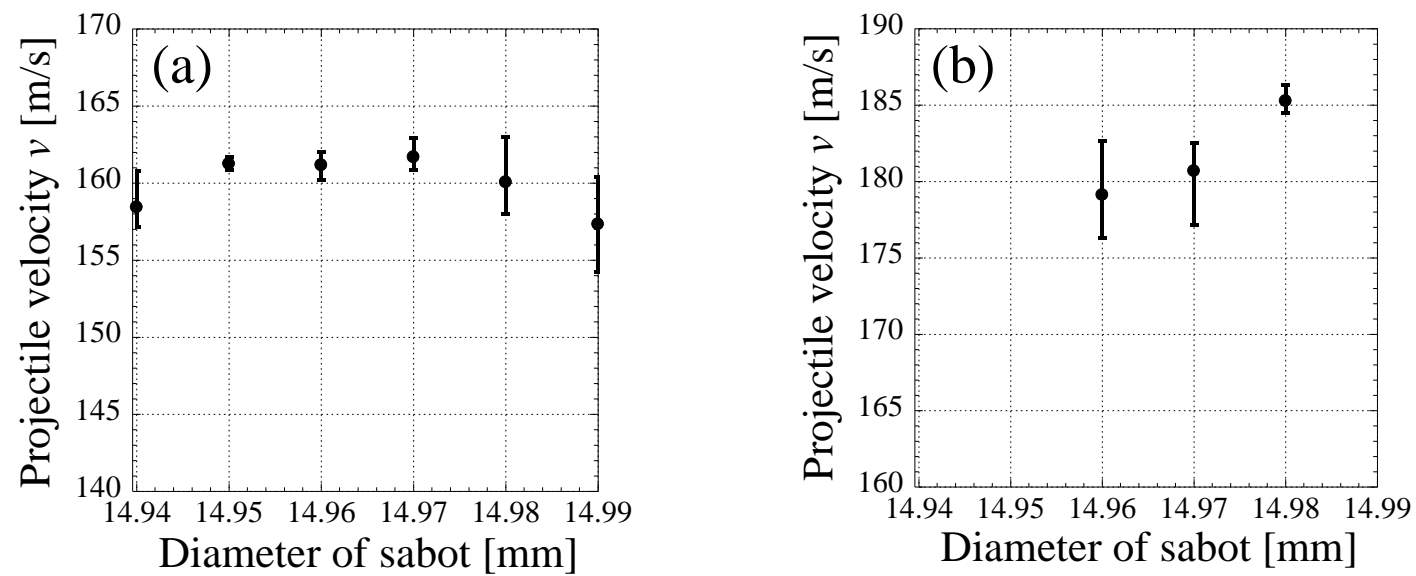

Fig. 8 Average experimental projectile velocities for $1 \mathrm{MPa}$ of (a) nitrogen and (b) helium gas.
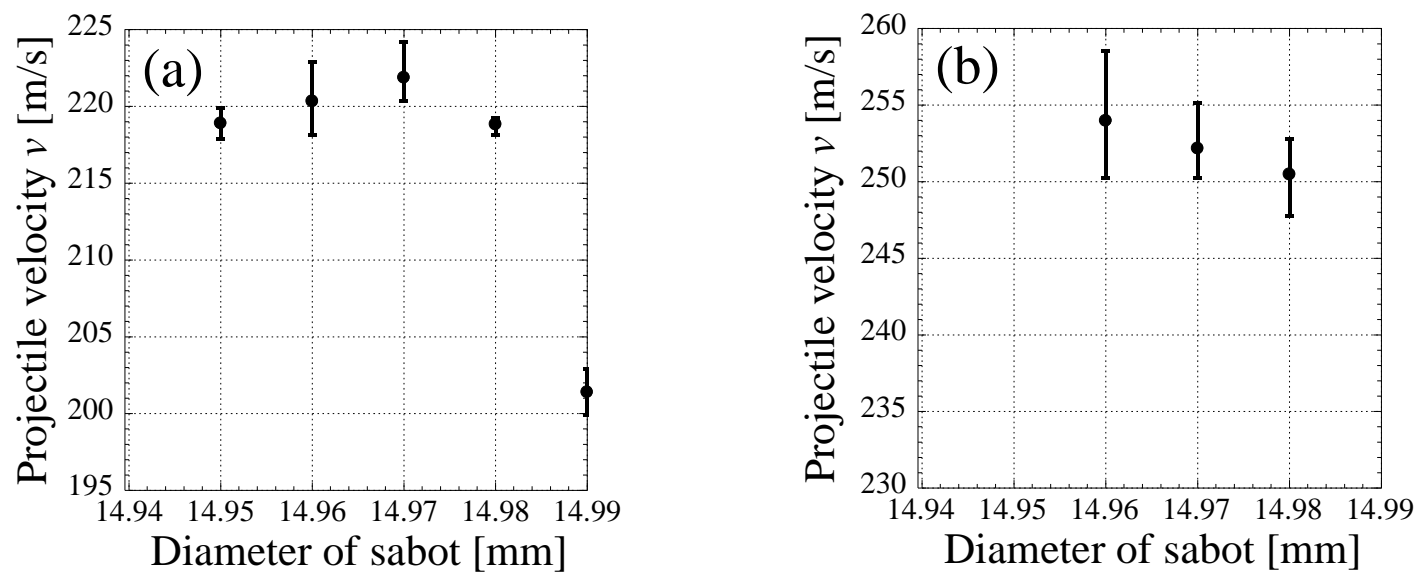

Fig. 9 Average experimental projectile velocities for $2 \mathrm{MPa}$ of (a) nitrogen and (b) helium gas.
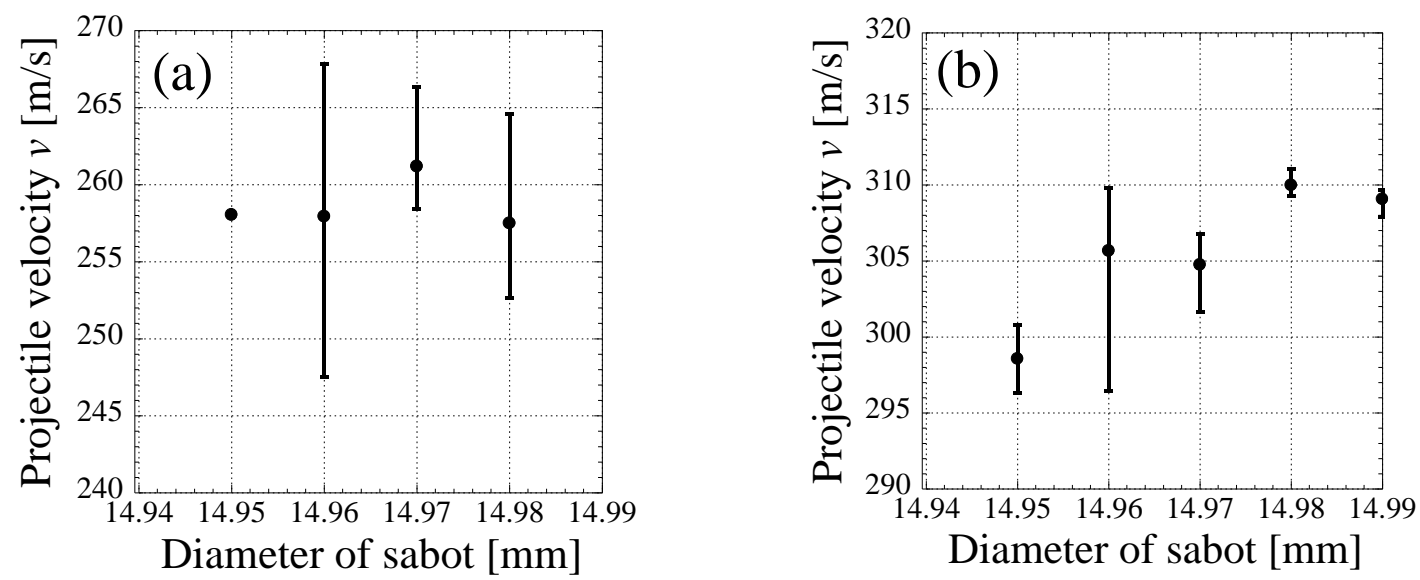

Fig. 10 Average experimental projectile velocities for $3 \mathrm{MPa}$ of (a) nitrogen and (b) helium gas. 

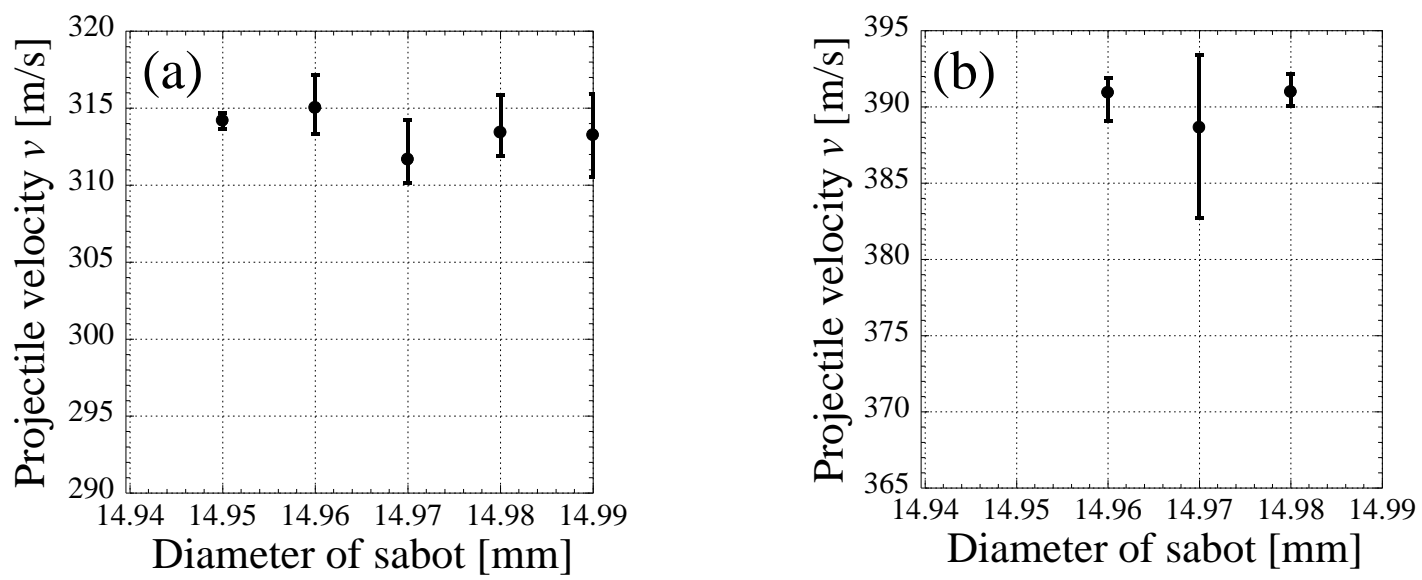

Fig. 11 Average experimental projectile velocities for $5 \mathrm{MPa}$ of (a) nitrogen and (b) helium gas.
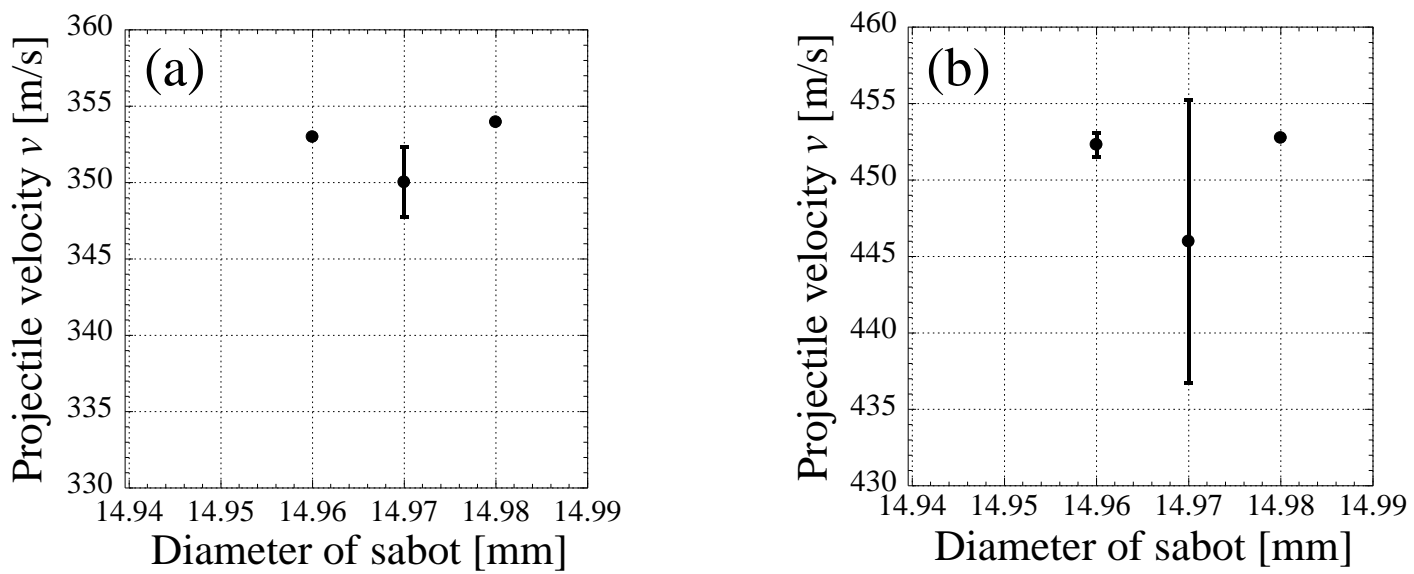

Fig. 12 Average experimental projectile velocities for $7 \mathrm{MPa}$ of (a) nitrogen and (b) helium gas.
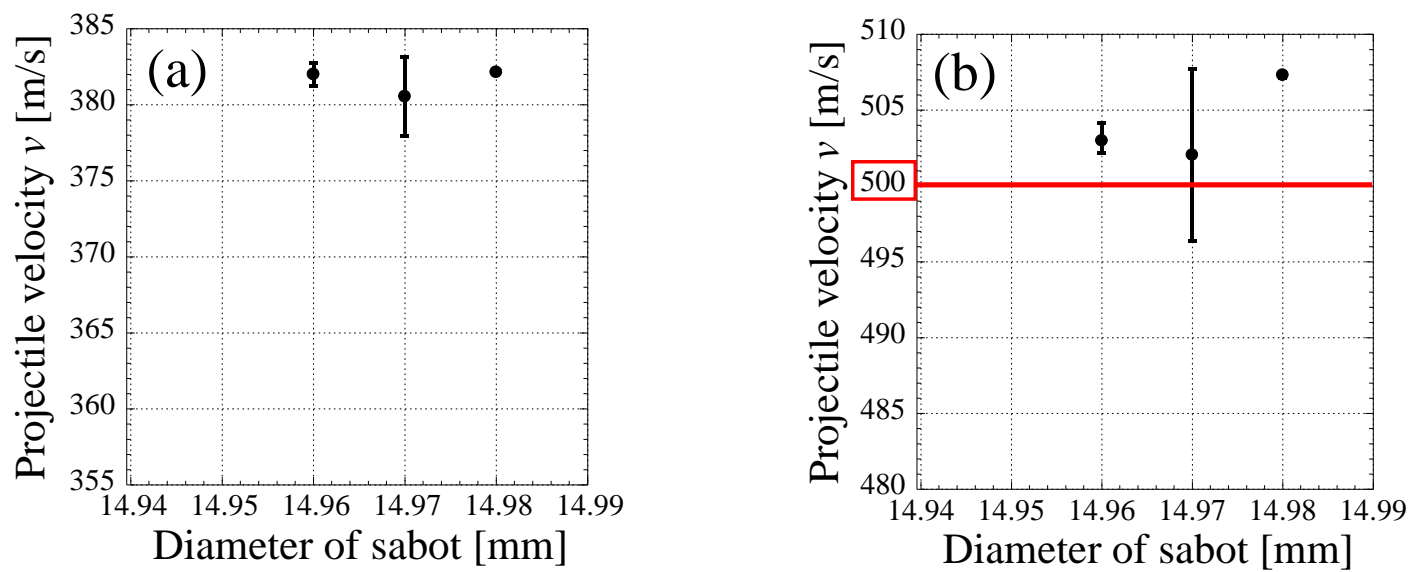

Fig. 13 Average experimental projectile velocities for $9 \mathrm{MPa}$ of (a) nitrogen and (b) helium gas. The red line indicates that the maximum target velocity was achieved with $9 \mathrm{MPa}$ of helium gas. 
First, experiments were conducted with $1 \mathrm{MPa}$ of nitrogen gas, shown in Fig. 8 (a). The results showed that the projectile velocity decreased for sabot diameters below $\phi 14.95 \mathrm{~mm}$, presumably caused by the leakage of propellant gas between the sabot diameter and the launch tube. Moreover, there was a decrease in velocity for sabot diameters of over $\phi 14.98 \mathrm{~mm}$, likely caused by frictional effects. A wide range of sabot diameters were also used for experiments with $2 \mathrm{MPa}$ of nitrogen gas (Fig. 9 (a)), $3 \mathrm{MPa}$ of helium gas (Fig. 10 (b)) and $5 \mathrm{MPa}$ of nitrogen gas (Fig. 11 (a)); the results of the experiments indicated that the projectile velocities were more or less within the same range. Regarding the placement of the projectile inside the launch tube, sabot diameters of $\phi 14.95 \mathrm{~mm}$ were usually found to be overly loose and sometimes required adhesive to place the projectile in the desired position. On the other hand, sabot diameters of $\phi 14.99 \mathrm{~mm}$ were usually too tight to allow the projectile to move through the launch tube; hence, further experiments were conducted using sabot diameters in the range of $\phi 14.96-14.98 \mathrm{~mm}$.

The velocity error calculations are given in Table 2. These data show that the total velocity error for each gas pressure was mostly in the $2 \%$ range, demonstrating that the gas gun investigated in this study is capable of consistently achieving projectile velocities with great accuracy. In addition, producing projectile sabots of diameters with error tolerances in the $1 / 100 \mathrm{~mm}$ range is an incredibly difficult task, and these experimental results show that projectile sabots can be produced with error tolerances in the $3 / 100 \mathrm{~mm}$ range, without causing a significant change in velocity. The experimental results were also compared with the theoretical velocity curve, as shown in Fig. 14, which illustrates the similar trends of the experimental results and the theoretical formula for both nitrogen and helium gases.

Table 2 Velocity error and average velocities of nitrogen and helium gas.

\begin{tabular}{|c|c|c|c|c|c|c|}
\hline $\mathrm{N}_{2}$ & $\begin{array}{c}1 \mathrm{MPa} \\
\text { Fig. } 7 \text { (a) }\end{array}$ & $\begin{array}{c}2 \mathrm{MPa} \\
\text { Fig. } 8 \text { (a) }\end{array}$ & $\begin{array}{c}3 \mathrm{MPa} \\
\text { Fig. } 9(\mathrm{a})\end{array}$ & $\begin{array}{c}5 \mathrm{MPa} \\
\text { Fig. } 10 \text { (a) }\end{array}$ & $\begin{array}{c}7 \mathrm{MPa} \\
\text { Fig. } 11(\mathrm{a})\end{array}$ & $\begin{array}{c}9 \mathrm{MPa} \\
\text { Fig. } 12 \text { (a) }\end{array}$ \\
\hline \multirow{2}{*}{$\begin{array}{c}\phi 14.96 \mathrm{~mm} \\
\text { Velocity error [\%] }\end{array}$} & +0.51 & +1.16 & +3.68 & +0.66 & ----- & +0.19 \\
\hline & -0.58 & -0.99 & -4.04 & -0.59 & ----- & -0.19 \\
\hline Average velocity [m/s] & 161.17 & 220.35 & 257.94 & 315.03 & 352.98 & 381.99 \\
\hline \multirow{2}{*}{$\begin{array}{c}\phi 14.97 \mathrm{~mm} \\
\text { Velocity error [\%] }\end{array}$} & +0.78 & +1.04 & +1.93 & +0.81 & +0.65 & +0.68 \\
\hline & -0.51 & -0.68 & -1.64 & -0.49 & -0.65 & -0.68 \\
\hline Average velocity [m/s] & 161.68 & 221.87 & 261.18 & 311.66 & 350.02 & 380.54 \\
\hline \multirow{2}{*}{$\begin{array}{c}\phi 14.98 \mathrm{~mm} \\
\text { Velocity error [\%] }\end{array}$} & +1.81 & +0.20 & +2.66 & +0.78 & ----- & ----- \\
\hline & -1.30 & -0.31 & -1.90 & -0.49 & ----- & ----- \\
\hline Average velocity $[\mathrm{m} / \mathrm{s}]$ & 160.06 & 218.83 & 257.51 & 313.41 & 353.96 & 382.14 \\
\hline He & $\begin{array}{c}1 \mathrm{MPa} \\
\text { Fig. } 7 \text { (b) }\end{array}$ & $\begin{array}{c}2 \mathrm{MPa} \\
\text { Fig. } 8(\mathrm{~b})\end{array}$ & $\begin{array}{c}3 \mathrm{MPa} \\
\text { Fig. } 9 \text { (b) }\end{array}$ & $\begin{array}{c}5 \mathrm{MPa} \\
\text { Fig. } 10(\mathrm{~b}) \\
\end{array}$ & $\begin{array}{c}7 \mathrm{MPa} \\
\text { Fig. } 11(\mathrm{~b}) \\
\end{array}$ & $\begin{array}{c}9 \mathrm{MPa} \\
\text { Fig. } 12(\mathrm{~b}) \\
\end{array}$ \\
\hline \multirow{2}{*}{$\begin{array}{c}\phi 14.96 \mathrm{~mm} \\
\text { Velocity error [\%] }\end{array}$} & +0.23 & +1.77 & +1.32 & +0.24 & +0.17 & +0.23 \\
\hline & -0.16 & -1.46 & -3.02 & -0.47 & -0.17 & -0.16 \\
\hline Average velocity [m/s] & 179.13 & 253.98 & 305.67 & 390.94 & 452.31 & 502.97 \\
\hline \multirow{2}{*}{$\begin{array}{c}\phi 14.97 \mathrm{~mm} \\
\text { Velocity error [\%] }\end{array}$} & +1.12 & +1.16 & +0.66 & +1.20 & +2.02 & +1.12 \\
\hline & -1.13 & -0.77 & -1.02 & -1.52 & -2.07 & -1.13 \\
\hline Average velocity $[\mathrm{m} / \mathrm{s}]$ & 180.69 & 252.16 & 304.74 & 388.64 & 445.98 & 502.05 \\
\hline \multirow{2}{*}{$\begin{array}{c}\phi 14.98 \mathrm{~mm} \\
\text { Velocity error [\%] }\end{array}$} & +0.04 & +0.91 & +0.35 & +0.31 & ----- & +0.04 \\
\hline & -0.04 & -1.09 & -0.29 & -0.23 & ----- & -0.04 \\
\hline Average velocity [m/s] & 185.28 & 250.49 & 309.97 & 390.97 & 452.76 & 507.30 \\
\hline
\end{tabular}



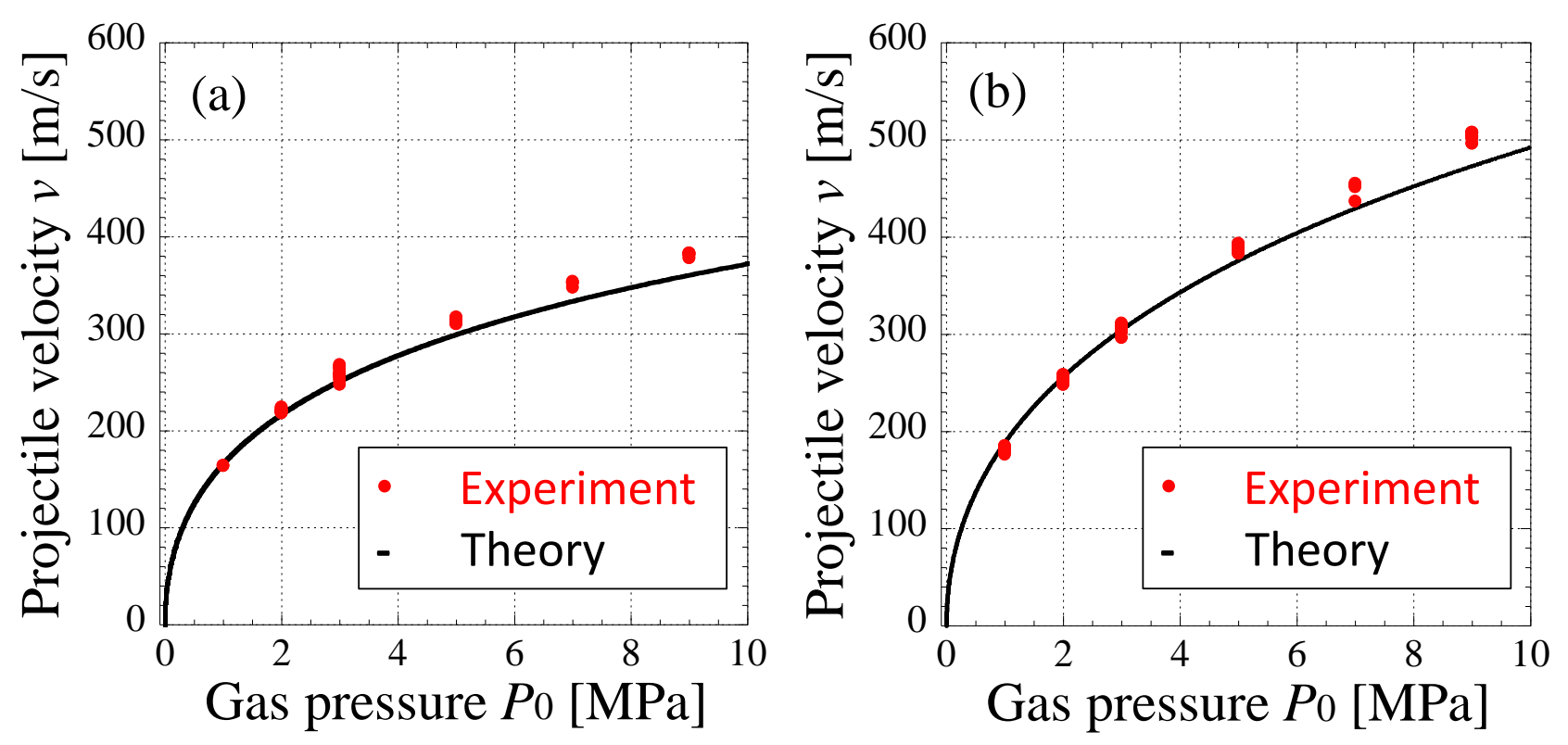

Fig. 14 Comparison between experimental results and the theoretical velocity curve for (a) nitrogen and (b) helium gas. All results are plotted for projectile sabot diameters of $\phi 14.96-14.98 \mathrm{~mm}$.

A precise prediction of the projectile velocity requires fitting the theoretical curve with the experimental results. To adjust the theoretical formula, fitting parameters $\alpha, \beta$ were proposed, as shown in Eq. (4). The $\alpha, \beta$ parameters are introduced as coefficients for the projectile velocity $v$ (on the $y$-axis of the curve) and the gas pressure $P_{0}$ (on the $x$-axis of the curve) to make appropriate corrections to the theoretical curve. The parameters in large brackets are also adjusted as a whole with the $\alpha$ parameter to finalize the adjustment. Equation (4) is plotted with the experimental results in Fig. 15, which clearly shows that the adjusted theoretical curve is in good agreement with the experimental results. The fitting parameters are listed in Table 3.
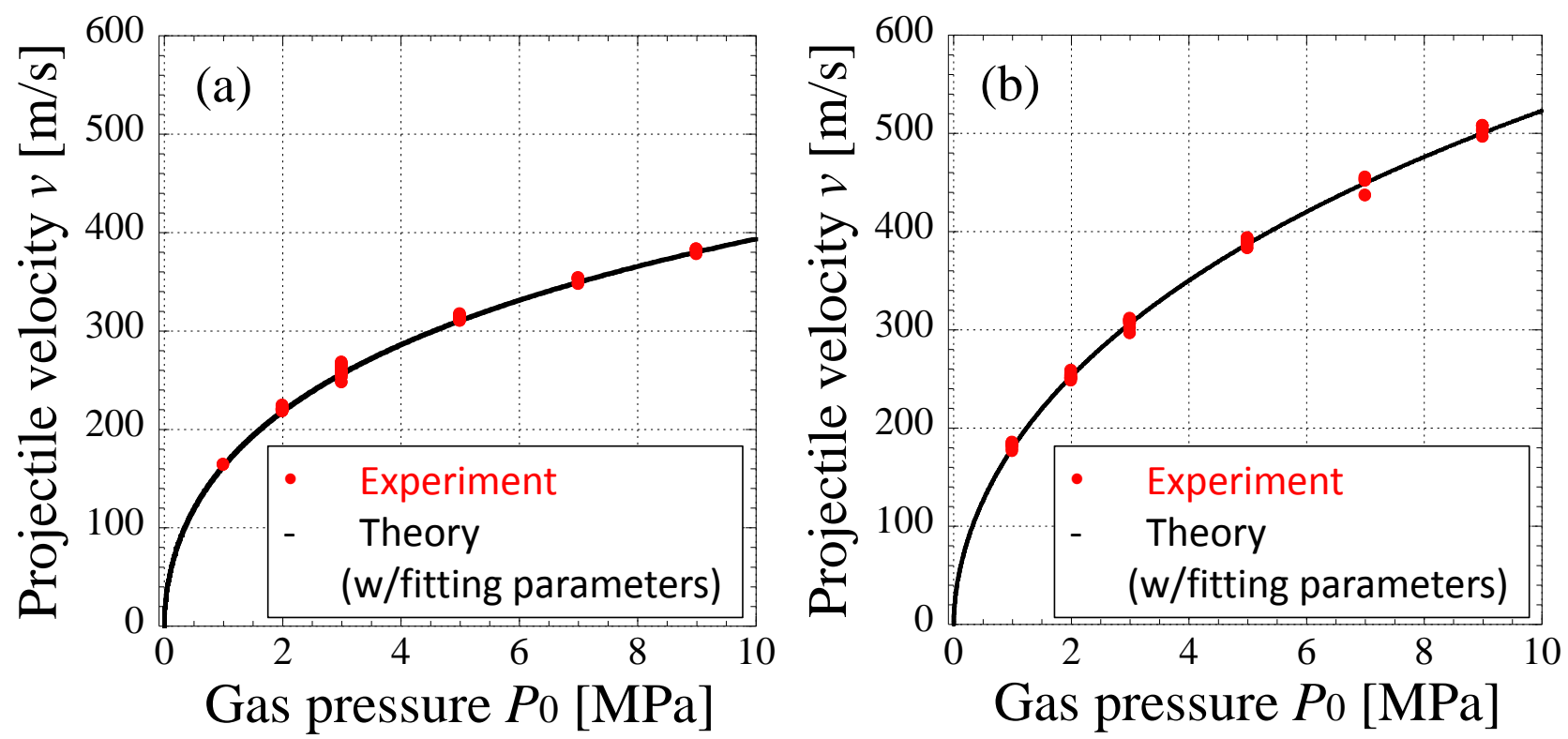

Fig. 15 Comparison between the experimental results and the theoretical velocity curve with the proposed fitting parameters for (a) nitrogen and (b) helium gas. 


$$
\beta P_{0}=\frac{2 m a_{0}^{2}}{A x(\gamma+1)}\left[\frac{\frac{2}{\gamma-1}-\frac{\gamma+1}{\gamma-1}\left\{1-\frac{\alpha v(\gamma-1)}{2 a_{0}}\right\}}{\left\{1-\frac{\alpha v(\gamma-1)}{2 a_{0}}\right\}^{\frac{\gamma+1}{\gamma-1}}}+1\right]^{\alpha}
$$

Table 3 Fitting parameters for nitrogen and helium gas.

\begin{tabular}{c|c|c}
\hline \hline Fitting parameters & $\mathrm{N}_{2}$ & $\mathrm{He}$ \\
\hline$\alpha$ & 0.94 & 0.90 \\
\hline$\beta$ & 0.94 & 1.02 \\
\hline
\end{tabular}

\section{Conclusions}

The performance evaluations conducted in this study indicate the following conclusions.

(1) The maximum target velocity of around $500 \mathrm{~m} / \mathrm{s}$ was achieved with $9 \mathrm{MPa}$ of helium gas.

(2) The experimental results showed that the projectile velocity is controllable with minimal velocity error, and guaranteed performance is attainable with projectile sabot diameters of $\phi 14.96-14.98 \mathrm{~mm}$.

(3) The experimental results showed trends similar to the theoretical formula. To predict the projectile velocity for all pressure ranges, fitting parameters were introduced to the theoretical formula, and the newly adjusted theoretical formula showed good agreement with the experimental results.

\section{Acknowledgements}

This work was supported by JSPS KAKENHI Grant Number 26289150.

\section{References}

Doolan, C., A two-stage light gas gun for the study of high speed impact in propellants, DSTO Report (2001), pp.2-6. Moradi, A. and Khodadadiyan, S., Study of real gas behavior in a single-stage gas gun, International Journal of Mechanical, Aerospace, Industrial, Mechatronic, and Manufacturing Engineering, Vol.5, No.6 (2011), pp.948-952. Mueller, A. C. and Fernando, E. M., The dynamics of projectiles launched by a two-stage light-gas gun, DTIC Report (1991), AD-A274 380, pp.1-8.

Putzar, R. and Schaefer, F., EMI's twin gun concept for a new light-gas gun type hypervelocity accelerator, Procedia Engineering, Vol.103 (2015), pp.421-426.

Rahner, C., Al-Qureshi, H. A., Stainer, D., Hotza, D. and Fredel, M. C., Numerical evaluation of a light-gas gun facility for impact test, Modelling and Simulation in Engineering, Vol.2014 (2014), Article ID 501434, pp.1-6.

Seigel, A. E., The theory of high speed guns (1965), pp.7-22, North Atlantic Treaty Organization Advisory Group for Aerospace Research and Development.

Thompson, P. A., Compressible Fluid Dynamics (1972), p.640, McGraw-Hill. 